\title{
The Scale and Characteristics Strength of SURF Feature Points Adaptive Matching Algorithm
}

\author{
Xiaotong Hu , Hui Ren ${ }^{\mathrm{b}}$ and Nan $\mathrm{Liu}^{\mathrm{c}}$ \\ Tianjin University of Science \& Technology, Tianjin 300000, China; \\ ahuxiaot@163.com, b826309057@qq.com, ‘819121957@qq.com
}

Keywords: Image registration, SURF, scale, feature point.

\begin{abstract}
In order to improve the matching precision of the SURF image feature points, we study the relationship among the scale of the SURF feature points, the characteristics of strength and matching precision. Then, a feature point matching algorithm of SURF image feature is proposed, which is adaptive to the feature point scale and characteristic strength. On the basis research of the scale and characteristics strength of SURF feature points, we found that the larger the scale of feature points set, the lower the matching precision will be; and the higher the intensity of characteristic, the matching precision are higher, but the number is less. Therefore, the using of the adaptive algorithm which can match the different scales of feature points with different characteristics strength threshold can keep the balance between the feature point matching precision and the quantity of matching feature points pairs, and realize the high precision of SURF feature points matching. The experimental shows that, compared with the traditional algorithm of SURF, this algorithm can obtain more precise matching feature points pairs.
\end{abstract}

\section{Introduction}

Image registration is a fundamental problem in the field of machine vision, and it is always the hot and difficult spot research. The method based on feature point registration is the first to extract features from the original image, and then establish the relationship between the two images, and this method has higher robust. SURF algorithm was proposed by Bay et al. [1], it is an improvement on the SIFT algorithm, which performance exceeds the SIFT and can achieve faster speeds [2]. The literature [3] proposed that SURF algorithm is the most robust performance local characteristics algorithm based on the performance evaluation of the typical local feature algorithm.

In this paper, we based on the characteristics of SURF feature points scale size, first to extract the small scale feature points of image to do the traditional matching, then set the large scale feature point constraint, and based on the feature point matching's results to estimate the image registration parameters, which make the detected image rotate to the standard image location accurately to achieve the purpose of registration.

\section{Detection And Description of SURF Feature Point}

SURF feature points matching algorithm not only has good robust about the image rotation, translation, scaling and noise, but also can deal with the change of light, invariance of view change and ambiguity of image.

\subsection{Detection of Feature Point}

SURF is a feature point detection method based on scale space. Firstly Gaussian smoothing to the image, and establish Gaussian scale space, then determine the location of the feature points by calculating the local extremum of Hessian matrix determinant. In the space, for any point $\hat{x}=(x, y)$ in the Hessian matrix, which scale is $\sigma$ can defined as:

$$
H=\left[\begin{array}{cc}
L_{x x}(\hat{x}, \sigma) & L_{x y}(\hat{x}, \sigma) \\
L_{x y}(\hat{x}, \sigma) & L_{y y}(\hat{x}, \sigma)
\end{array}\right]
$$


In the formula, ${ }^{L_{x x}}$ is a convolution result of the Gaussian second derivative $\frac{\partial^{2}}{\partial x^{2}} g(\sigma)$ with $I=(x, y)$, among them $g(\sigma)=\frac{1}{2 \pi \sigma^{2}} e^{-\left(x^{2}+y^{2}\right) / 2 \sigma^{2}} . L_{x y}, L_{y y}$ Have the same meaning.

\subsection{Description of The Feature Points}

In order to maintain the rotation invariance of feature points, we should determine the main direction for each feature points after getting the location of feature point. Therefore, setting the feature point as the center, in the circular area which radius is $6 \sigma$ ( $\sigma$ is the scale of the feature points) execute the Harr wavelet(length $4 \sigma$ ) response operation on the $\mathrm{x}$ and y direction of the image and use the Gaussian weighting function which scale is $2 \sigma$ to weighted the Harr wavelet response value in order to make the closer to the feature points, the greater contribution; Then use the fan-shaped area which size is $\pi / 3$ to traverse the entire circle area, and set the direction of the maximum value of the vector and die of magnitude of the horizontal and vertical response as the main direction of the feature points.

Then, with a feature point as the center, construct a square window area that one side perpendicular to the main direction and the side with length of $20 \sigma$, and divided the window area into the $4 \times 4$ subdomains. In each subdomain, calculate the horizontal and vertical direction Harr wavelet response which has $5 \sigma \times 5 \sigma$ sample points, denoted by $d_{x}$ and $d_{\mathrm{y}}$ respectively. As the same time, use the Gaussian weighting function which scale is $2 \sigma$ to weight the Harr wavelet response. Then add the response value and the absolute value of each subdomain. As a result, each subdomain has formed a 4-dimensional characterization vectors:

$$
V=\left(\Sigma d_{x}, \Sigma d_{x}, \Sigma\left|d_{x}\right|, \Sigma\left|d_{y}\right|\right)
$$

For each feature point there finally have formed $4 * 4 * 4=64$ - dimensional feature vector. In order to keep the illumination invariance, execute the normalization processing to each feature vector, and then get the ultimate SURF descriptor.

\subsection{Feature Point Matching}

The key to realize the image registration is the matching of feature points, the matching precision will directly affects the subsequent registration accuracy. This paper according to the feature point neighborhood information which SURF feature points descriptor contained, using the K-nearest neighbor algorithm to find out two potential best matching points of each feature points, then select the best matching points pairs by comparing the best and the second best distance value ratio.

Set $M_{1}, M_{2}$ with two images $I_{1}, I_{2}$,then SURF were used respectively to extract for matching feature points, and any feature point $m_{1 i}$ in $M_{1}$, the two feature points $m_{2 \mathrm{j}}, m^{\prime}{ }_{2 \mathrm{j}}$ which have the minimum Euclidean distance with $m_{1 i}$ in $M_{2}$, the distance respectively are $d_{i j}, d^{\prime}{ }_{i j}$. If $d_{i j} \leq \alpha * d^{\prime}{ }_{i j}$, then we think $m_{1 i}$ and $m_{2 \mathrm{j}}$ is the optimization matching points pair.

\section{The Influence Factors of The Matching Precision}

\subsection{The Relationship between Feature Points Scale and Positioning Accuracy.}

According to the detection and description of SURF feature points, the sale of the feature points is important characteristic, at the same time it also reflects the neighborhood information which is the basis for matching feature points. As is shown in figure 1. 


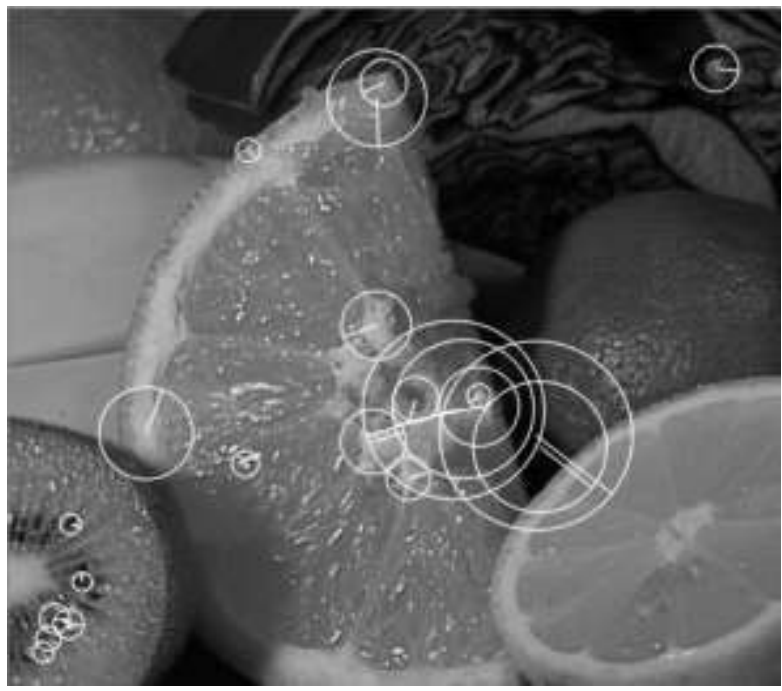

Fig. 1 The scale of SURF feature points

In the process of image registration based on feature points, the matching of feature points affects the estimation of the parameters of the image registration transformation model. How to select the high accuracy matching feature points is the key to improve the accuracy of image registration. According to the relationship of the feature points, the small scale feature points are less affected by the neighborhood information which is possible to describe the information more accurately So that the matching between the feature points has a high accuracy [4]. So it can be assumed that the small scale feature points can improve the accuracy of image registration.

In order to demonstrate the effect of feature point scale, we make some analyzes on image registration. Under the conditions of the image rotation angle (Experiment 5 degrees), we according to the known angles calculate the rotated actual coordinates of the matched feature points, and differentially calculate the coordinate values with the matched feature points on the Pending image. The absolute values of the positioning error of the characteristic points shown in Figure 2 are obtained.

From Figure 2, it can be seen that under the rotating condition, the small scale feature points generally have the characteristic which have the small difference with the actual coordinate value. It can be suspected that the feature points can effectively improve the accuracy of feature points matching.

\subsection{The Relationship between Feature Intensity and Positioning Accuracy.}

As a result of this article is based on the feature point neighborhood information contained by the SURF feature point descriptor, use the K nearest neighbor method to identify the two best match point of each feature point potential, and select the best matching points by the best value of the distance of the matching points and the ratio of the second best value [5]. So we can consider that controlling the detection accuracy of feature points by adjusting the ratio of alpha value.

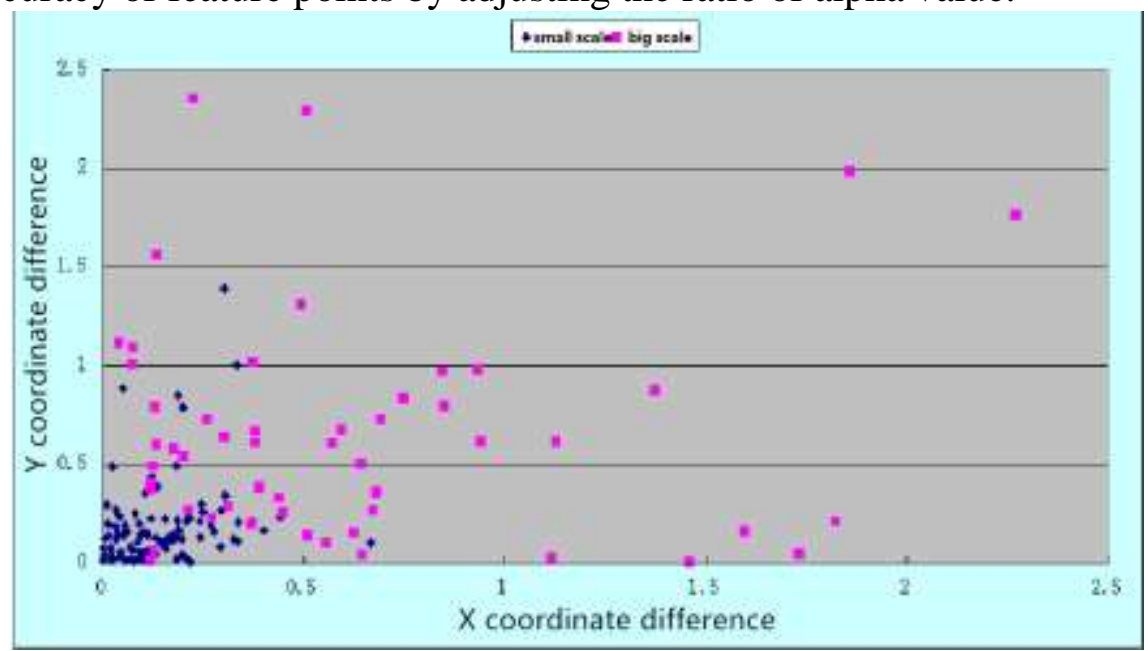

Fig. 2 Coordinate difference results of different scale feature points in rotating condition 
In this regard, we definite the Euclidean distance d1 of the eigenvector of the feature with the highest similarity to be matched and the Euclidean distance $\mathrm{d} 2$ of the eigenvector of the feature point to be matched 's radio $\mathrm{d} 1 / \mathrm{d} 2$ is characteristic strength of the feature points. Because the higher the similarity, the Euclidean distance is smaller, the ratio of the Euclidean distance of the characteristic point with the same degree of similarity - the smaller the characteristic intensity value.

In order to study the relationship between the feature intensity threshold and the accuracy of the feature points, we analyze the error of the rotating image with different intensity threshold. After 10 degree rotation of the image, the feature points of the image are matched with the images before.

Subsequently, we calculate the rotation of the image feature points by 10 degree rotation coordinates, and calculate the $\mathrm{X}$ and $\mathrm{Y}$ difference absolute value of the coordinates and rotated image matching feature point coordinates, then we get the positioning error of the feature points, the results as shown in Figure 3. At the same time, we set the threshold of different characteristic intensity, and the error of the positioning error is analyzed, and the characteristic is also analyzed.

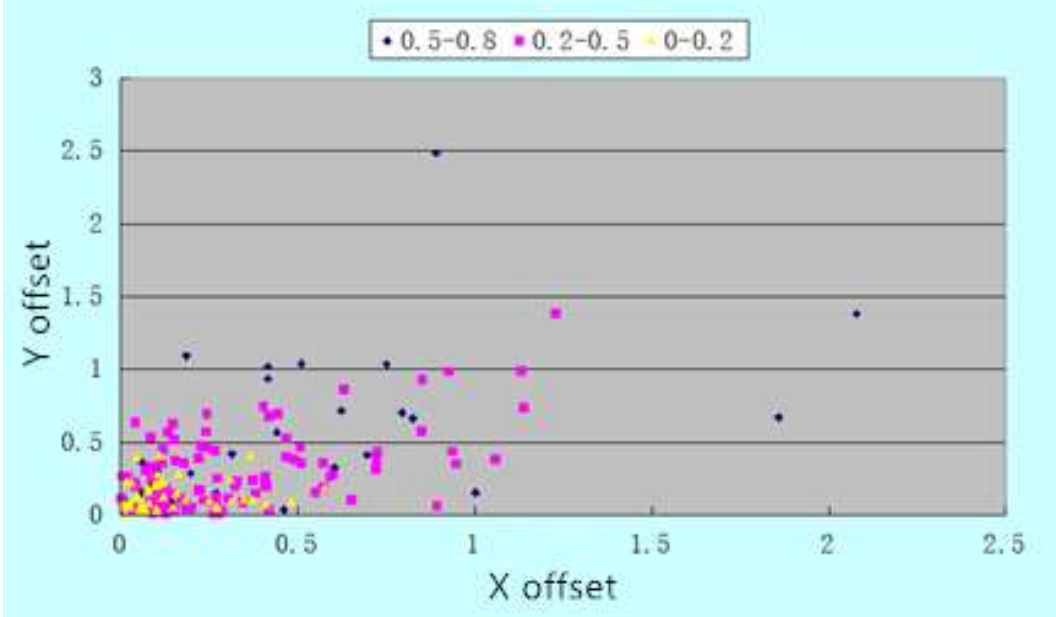

Fig. 3 Position error of different intensity feature points

From Figure 3, it can be seen that the error of the location error is gradually increasing with the increase of the threshold of the characteristic intensity, which indicates that the threshold value of the feature has a effect on the accuracy of the feature points.2.3 relationship between scale, characteristics of strength and location accuracy.

\subsection{The Relationship Between Scale, Feature Intensity and Location accuracy}

The relationship between the scale of SURF feature points, the feature points and the location accuracy of the feature points are discussed respectively in the 2.1 and 2.2 sections. From the results of the experiment, we can see that: with the increase of scale, the positioning accuracy of the feature points decreases, with the increase of the feature intensity, the positioning accuracy of the feature points decreases.

Traditional SURF feature points matching algorithm uses uniform intensity threshold, when the threshold set is small, it may cause a part of the positioning accuracy of small scale feature are filtered; and when the threshold value is set too large, it may make lower part positioning accuracy of large scale feature points be preserved. So in this paper, a characterization of uniform intensity threshold may influence the overall positioning accuracy, or reduce successful matching feature points on the number, and become hard to achieve the matching accuracy and the matching of feature points among the quantities of balance.

A feature point matching algorithm for SURF with feature points and feature intensity adaptive is proposed. The algorithm uses the large feature intensity threshold for the small scale feature points with higher accuracy to retain more small scale feature points; for the positioning accuracy which is not of high large scale feature, using the characteristic of small intensity threshold, to eliminate low positioning precision of large scale feature. Using the above method, the feature points of the feature points can be obtained more successfully when the feature points are improved. 


\section{The Experimental Results}

\subsection{Relationship Between Scale and Accuracy}

In order to quantitatively analyze the relationship between the feature points and the accuracy, the relationship between the scale and the accuracy of the feature points is studied. Rotate the two test images by 10 degrees to get the image to be matched. In the same characteristic intensity (Experiment from the intensities of the characteristic threshold of 0.5), the SURF feature points matching image and original image will be matching calculated. The locating error of the feature points of the matching is calculated, and the location accuracy of the characteristic points of different scales is analyzed. Positioning error calculation method will select the image to match the success of feature points and the original in the corresponding feature points matching and the coordinates of the relative displacement among 10 degrees rotation of coordinates.

Table 1. Average positioning error of different scale feature points

\begin{tabular}{cccc}
\hline image & scale & $\begin{array}{c}\text { The number of } \\
\text { feature points }\end{array}$ & $\begin{array}{c}\text { A verage position error } \\
\text { (pixels) }\end{array}$ \\
\hline \multirow{3}{*}{ Image1 } & size $<20$ & 124 & 0.4373 \\
& $20<$ size $<40$ & 127 & 0.5834 \\
& size $>40$ & 65 & 1.1677 \\
Image2 & size $<20$ & 258 & 0.4139 \\
& $20<$ size $<40$ & 174 & 0.6217 \\
& size $>40$ & 67 & 1.1677 \\
\hline
\end{tabular}

From table 1, the average position error of the feature points is increased with the increase of the feature points. At the same time, different scales of feature points between the positioning error difference is larger, such as the average positioning error of the feature points with a scale greater than 40 is close to three times the positioning error of the feature points with dimensions less than 20.

\subsection{Relationship Between Feature Intensity and Location accuracy}

In order to analyze the relationship between the feature intensity and the accuracy, the experiment of the relationship between the feature intensity and the accuracy of the location is studied. Two degrees of image rotation are 10 degrees respectively, and then the image is to be matched. In the same scale (the size of less than 40), the SURF feature point matching image and original image will be matching calculated. The locating error of the feature points of the matching is calculated about the matched successful feature points, and the location accuracy of the characteristic points of different characteristic intensities is analyzed. Among them, the calculation method of location error is the same as 3.1 .

Table 2. Average error of different intensity feature points

\begin{tabular}{cccc}
\hline image & Characteristic strength & $\begin{array}{c}\text { The number of } \\
\text { feature points }\end{array}$ & $\begin{array}{c}\text { A verage position error } \\
\text { (pixels) }\end{array}$ \\
\hline \multirow{2}{*}{ Image1 } & $\alpha<0.2$ & 78 & 0.3359 \\
& $0.2<=\alpha<0.5$ & 176 & 0.6107 \\
$0.5<=\alpha<0.8$ & 58 & 1.0968 \\
$\alpha<=0.2$ & 127 & 0.3016 \\
Image2 & $0.2<\alpha<=0.5$ & 317 & 0.5653 \\
& $0.5<\alpha<=0.8$ & 92 & 0.8515 \\
\hline
\end{tabular}

From the table 2, the average position error of the feature increases with the increase of the characteristic intensity. At the same time, the positioning error characteristic differences among different characteristics of intensity is large, such as the average positioning error of the feature points with characteristic intensities between 0.5 and 0.8 is close to three times the positioning error of the feature points with characteristic intensities less than 0.2.

\subsection{Relationship Between Scale, Feature Intensity and Accuracy of Location}

As above, the accuracy of feature points matching can be improved effectively by using small scale feature points or improving the feature intensity. In order to study the relationship among the three further, we extract feature points in different scale and rotate the image, and calculate the average migration distance according to the characteristics of different intensity, as shown in Table 3 results. 
From table 3 we can see that smaller the feature point scale and the more obvious features of strength, the smaller the average migration distance of match point, the higher the precision. In most of the time, we need to have a large number of points within a specific accuracy (where the average offset is less than 1), relying on unilateral factors cannot meet the needs of the number and accuracy, therefore, we consider the feature point of size $<20$ to match with $0<\alpha<0.8$ characteristic intensity, the feature point of size $>40$ to match with $0<\alpha<0.2$ characteristic intensity. And then, with the matching accuracy we get more the matching point.

\subsection{Comparison With The Traditional Surf Algorithm}

Table 3. Average positioning error of feature points under different scales and feature intensities

\begin{tabular}{|c|c|c|c|c|}
\hline image & scale & $\begin{array}{c}\text { Characteristic } \\
\text { strength }\end{array}$ & $\begin{array}{l}\text { The number of } \\
\text { feature points }\end{array}$ & $\begin{array}{l}\text { A verage position error } \\
\text { (pixels) }\end{array}$ \\
\hline \multirow{10}{*}{ Image 1} & \multirow{3}{*}{ size $<20$} & $\alpha<0.2$ & 71 & 0.2679 \\
\hline & & $0.2<\alpha<0.5$ & 176 & 0.5245 \\
\hline & & $0.5<\alpha<0.8$ & 66 & 0.8263 \\
\hline & \multirow{3}{*}{$20<$ size $<40$} & $\alpha<0.2$ & 52 & 0.4183 \\
\hline & & $0.2<\alpha<0.5$ & 129 & 0.9023 \\
\hline & & $0.5<\alpha<0.8$ & 40 & 1.3166 \\
\hline & \multirow{4}{*}{ size $>40$} & $\alpha<0.2$ & 27 & 0.9589 \\
\hline & & $0.2<\alpha<0.5$ & 40 & 1.4739 \\
\hline & & $0.5<\alpha<0.8$ & 12 & 1.9545 \\
\hline & & $\alpha<0.2$ & 65 & 0.2755 \\
\hline \multirow{8}{*}{ Image 2} & \multirow[t]{2}{*}{ size $<20$} & $0.2<\alpha<0.5$ & 177 & 0.4697 \\
\hline & & $0.5<\alpha<0.8$ & 49 & 0.6369 \\
\hline & & $\alpha<0.2$ & 61 & 0.4223 \\
\hline & \multirow[t]{3}{*}{$20<\operatorname{size}<40$} & $0.2<\alpha<0.5$ & 99 & 0.7496 \\
\hline & & $0.5<\alpha<0.8$ & 23 & 1.3886 \\
\hline & & $\alpha<0.2$ & 25 & 0.9279 \\
\hline & \multirow[t]{2}{*}{ size $>40$} & $0.2<\alpha<0.5$ & 37 & 1.2871 \\
\hline & & $0.5<\alpha<0.8$ & 8 & 1.3735 \\
\hline
\end{tabular}

In order to verify the effectiveness of the algorithm, the feature points matching experiment is carried out by using the traditional SURF algorithm and the adaptive algorithm proposed in this paper. At the same time, in order to accurately compare the methods, we used different feature intensity threshold in the traditional method of experimental so that the number of matching pairs of features and the average positioning error are similar to those of the algorithm in order to analyze experimental results of the two methods more accurately.

Table 4. The comparison of the matching accuracy between the proposed and the traditional SURF algorithm

\begin{tabular}{ccccccc}
\hline & \multicolumn{2}{c}{ Proposed method } & \multicolumn{4}{c}{ Traditional method } \\
\cline { 2 - 7 } image & $\begin{array}{c}\text { The } \\
\text { numberof } \\
\text { matching } \\
\text { points }\end{array}$ & $\begin{array}{c}\text { Average } \\
\text { position } \\
\text { error (pixels) }\end{array}$ & $\begin{array}{c}\text { Low matching accuracy } \\
\text { settings } \\
\text { The number } \\
\text { of matching } \\
\text { points }\end{array}$ & $\begin{array}{c}\text { Average } \\
\text { position } \\
\text { error (pixels) }\end{array}$ & $\begin{array}{c}\text { The number } \\
\text { of matching } \\
\text { points }\end{array}$ & $\begin{array}{c}\text { Average } \\
\text { position } \\
\text { error } \\
\text { (pixels) }\end{array}$ \\
\hline Image1 & 354 & 0.6380 & 352 & 0.7128 & 299 & 0.6345 \\
Image2 & 565 & 0.5460 & 565 & 0.6352 & 346 & 0.5492 \\
Image3 & 637 & 0.6456 & 639 & 0.7227 & 508 & 0.6459 \\
\hline
\end{tabular}

From table 4, we can see that the characteristic of the traditional method could not achieve the balance for the successful feature points matching between the number and the average positioning error. That is to say, when getting a large number of matched point pairs, average positioning error is larger; when getting a smaller average positioning error, the number of feature points will decrease. Compared with the traditional SURF algorithm, the average localization error of the algorithm is reduced by $10 \%-15 \%$ when the matching accuracy is close; when the matching precision is similar, the number of feature points extracted by this algorithm is increased by $18 \%-63 \%$. From the above 
results, compared with the traditional SURF feature point matching algorithm, the matching feature points are more quantity and matching accuracy is higher.

\section{Conclusion}

In this paper we study the surf feature points and matching accuracy of both scale and strength characteristics of the relationship between found:

we study the relationship between the scale, feature intensity and matching accuracy of SURF feature points and find that with the increase of the scale, feature point positioning accuracy is decreased; with the increase of characteristic strength, feature point positioning accuracy also have showed a downward trend. So an adaptive image registration algorithm based on SURF feature points and feature intensity is proposed, which solves the problem that the single feature intensity threshold can't get more accuracy matching feature points. Experimental results show that the algorithm can effectively improve the accuracy of the feature points, and get more matching feature points.

\section{References}

[1]. BAY H, TUYTELAARS T, Van COOL L. Speeded-Up Robust Features (SURF). Computer Vision and Image Understanding. Vol. 110 (2008) No. 3, p. 346-359.

[2]. L Juan, O Gwun. A comparison of SIFT, PCA-SIF and SURF. International Journal of Image Processing. Vol. 3 (2009) No. 4, p. 143-152.

[3]. MIKOLAJCZYK K, SCHMID C. A performance evaluation of local descriptors. IEEE Trans on Pattern Analysis and Machine Intelligence. Vol. 27 (2005) No. 10, p.1615-1630.

[4]. Lowe, D.G. Distinctive Image Features from Scale-Invariant Keypoints. International Journal of Computer Vision. Vol. 60 (2004) No. 2, p. 91-110.

[5]. He Zhoucan, Wang Qin, Yang Heng. An Extended Local Sensitivity Hash Algorithm for Efficient Image Matching. Journal of Sichuan University: Natural Science Edition. Vol. 47 (2010) No. 2, p. $269-274$. 